\title{
VOCAL TRACT MORPHOLOGY IN INHALING SINGING: AN MRI BASED STUDY
}

\author{
M.B.J. Moerman1, F. Vanhecke2, L. Van Assche3, J. Vercuysse 3, K. Daemers4, M. 1
}

\section{Leman5}

Department of ENT/Phoniatrics and Head and Neck Surgery, Maria Middelares, Ghent, Belgium 2

University College, School of Arts, Ghent, Belgium

${ }_{3}$ Radiology Department, Maria Middelares, Ghent, Belgium 4 Speech andsLanguage Rehabilitation

Department, Maria Middelares, Ghent, Belgium

Musicology Department, Ghent University, Ghent, Belgium mmoerman @ skynet.be

Introduction: Inhaling singing is a recently developed singing technique explored by the soprano singer Françoise Vanhecke. It is based on an aspiratory airflow instead of an expiratory airflow. Based on the spectral analysis and on the fact that there is no audible difference between the tones sung by the in- or exhaling technique [1], we hypothesize that the vocal tract remains the major determinant of the resonance features. However, we expect the subglottal region to narrow in inhaling singing, secondary to the inverse airstream. Meanwhile we also question if the morphology of the supraglottal vocal tract would significantly alter.

Methods: A female professional singer and composer (second author) sung 6 prolonged tones starting at F5 and chromatically rising up to Bb5 on the vowel /a/ in a decline position under MRI, both in the exhaling and inhaling manner. All images were performed on a Philips 1.5 T Philips Achieva scanner (Philips Medical Systems, Best, The Netherlands) by using sensitivity-encoding 16 channel head and neck coil. This provided 12 midsaggital images (2X6). Various anatomical structures (distances, angles and areas) were measured.

Statistical analysis

Wilcoxon directional testing was performed on all measurements (Table 1 -not included due to limited length). Results: A statistically significant difference is found for the area of the complete vocal tract (features $17+18+19$ in table 1 ). The vocal tract is larger in exhaling singing compared to inhaling singing. Focusing on its components we notice that there is no statistically significant difference for the volume of the area of the anterior oral cavity (feature 17 in table 1), nor for the area of the oro-hypopharyngeal region (feature 18 in table 1) or the sum of both. The statistically significance is mainly due to a decrease of the subglottal area in the inhaling singing condition (feature 19 in table 1). There is also a statistically significant change in the tongue configuration (feature 3, 4, 5 in table 1), in the distance between the teeth (feature 1 in table 1), in the length of the floor of mouth (feature 14 in table 1), and in the position of the epiglottis (feature 10 and 11 in table 1). Discussion: The narrowing of the subglottis is considered to be secondary to suction forces used in the inhaling singing technique. Vanhecke et al. described a similar frequency distribution in the in- and exhaling condition, which concurs with an audible alikeness between in- and exhaling singing tones but they also state that the harmonic structure is less rich[1]. This suggests a similar vibration pattern at the glottal level and the role of the vocal tract as the most important resonator, albeit in a more rigid way. The preserved distance between the posterior tongue and posterior hypopharyngeal wall (feature 7 in table 1), the distance between the tongue dorsum and the palate (feature 8 in table 1) and the angle between the mandibulo-hyoidal line and the tongue thickness- line (feature 9 in table 1) indicate that the two major dimensions of the tongue positioning for the vowel /a/, namely back and low, do not differ in the two singing techniques. However, we observe a statistically significant decrease of the anterior-posterior diameter of the tongue, the tongue thickness and the tongue tip curvature (feature 1, 3, 4, 5 in table 1) in inhaling singing. These findings in contrast with the preserved vocal tract volume and the preserved place of articulation strengthen our presumption that the tongue plays a larger role than merely a resonance modulator. We suspect that the more vertical position of the epiglottis in inhaling singing suggests an adaption of the vocal tract to facilitate the airflow immediately above the glottis. The significant increase in the distance between the chin and prelaryngeal soft tissue, independently from the angle (features 14 and 15 in table 1), suggests that no laryngeal elevation takes place. Elongation of the submental muscles indicates a tension raise and hence a strengthening of the floor of the mouth which no doubt assists in the tongue positioning and morphology.

The vibratory pattern of the vocal folds is currently under research.

References

[1]Vanhecke F, Moerman M, Desmet F, Raes GW, Leman M. Inhaling Singing: a new vocal technique with remarkable properties. Journal of Voice. 2015; in review The full article of this manuscript is in print (Journal of Voice, 2015) 